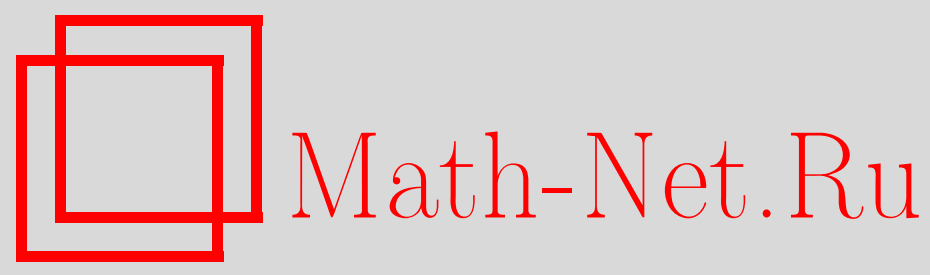

Ю. А. Коняев, Ю. С. Фёдоров, Асимптотический анализ некоторых классов сингулярно возмущенных задач на полуоси, Матем. заметки, 1997, том 62, выпуск 1, 111-117

DOI: https://doi.org/10.4213/mzm1593

Использование Общероссийского математического портала Math-Net.Ru подразумевает, что вы прочитали и согласны с пользовательским соглашением http://www . mathnet.ru/rus/agreement

Параметры загрузки:

IP : 3.89 .185 .249

26 апреля 2023 г., 16:14:25

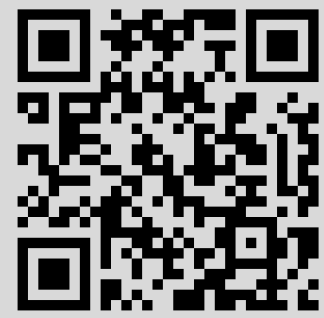




\title{
АСИМПТОТИЧЕСКИЙ АНАЛИЗ НЕКОТОРЫХ КЛАССОВ СИНГУЛЯРНО ВОЗМУШЕННЫХ ЗАДАЧ НА ПОЛУОСИ
}

\author{
Ю. А. Коняев, Ю. С. Фёдоров
}

\begin{abstract}
Предлагается новый метод асимптотического интегрирования некоторых классов сингулярно возмущенных начальных задач на полуоси для неоднородных систем линейных обыкновенных дифференциальных уравнений, что является развитием идей метода регуляризации.

Данный метод позволяет доказать существование единственного и ограниченного при $\varepsilon \rightarrow+0$ решения таких задач и указывает более простой и конструктивный алгоритм для построения асимптотического разложения решения с выделением всех его сингулярностей в замкнутой аналитической форме (вк лючая критический случай, когда точки спектра предельного оператора могут касаться мнимой оси), что дополняет или уточняет известные ранее результаты.

Библиограффия: 10 названий.
\end{abstract}

Предлагается новый метод асимптотического интегрирования некоторых классов сингулярно возмущенных начальных задач на полуоси $\mathbb{R}^{+}$для неоднородных систем линейных обыкновенных дифференциальных уравнений, что является развитием идей метода регуляризации [1].

В отличие от известного [2]-[5], данньй метод, используя результаты работ [6]-[9], позволяет доказать существование единственного и ограниченного при $\varepsilon \rightarrow+0$ решения указанных задач и предлагает более простой и конструктивньй алгоритм для построения асимптотического разложения решения с выделением всех его сингулярностей в замкнутой аналитической форме.

Рассмотрим на полуоси $\mathbb{R}^{+}$сингулярно возмущенную задачу Коши

$$
\varepsilon \frac{d y}{d x}=A(x, \varepsilon) y+f(x), \quad y(0, \varepsilon)=\alpha,
$$

где матричньй ряд $A(x, \varepsilon)=\sum_{0}^{\infty} A_{k}(x) \varepsilon^{k}$ сходится абсолютно и равномерно на полуоси $\mathbb{R}^{+}$при $|\varepsilon|<\varepsilon_{0}$, причем функции $f(x), A_{k}(x) \in C^{N+1}\left(\mathbb{R}^{+}\right)$ограничены на полуоси $\mathbb{R}^{+}$с нужньм количеством производных, т.е.

$$
\left\|f^{(p)}(x)\right\| \leqslant C_{p}, \quad\left\|A_{k}^{(p)}(x)\right\| \leqslant C_{k p}, \quad\left(p \in \overline{0, N+1}, \quad k \geqslant 0, \quad x \in \mathbb{R}^{+}\right),
$$

а матрица $A_{0}(x)$ имеет простой ненулевой стабильньй спектр $\left\{\lambda_{0 j}(x)\right\}_{1}^{n}$, т.е.

$$
\sigma_{j k}(x) \equiv \lambda_{0 j}(x)-\lambda_{0 k}(x) \neq 0, \quad\left|\lambda_{0 j}(x)\right| \geqslant \delta_{0}>0 .
$$


В отличие от сингулярно возмущенных начальных задач вида (1) на конечном отрезке, сингулярности решения аналогичной задачи на полуоси в общем случае будут определяться не только спектром матрицы $A_{0}(x)$, но и структурой некоторых интегралов, неограниченных при $x \rightarrow+\infty$.

Прежде чем сформулировать некоторые утверждения, получим сначала с помощью преобразования сдвига

$$
y(x, \varepsilon)=q(x, \varepsilon)+w_{(N)}(x, \varepsilon) \quad\left(w_{(N)}(x, \varepsilon)=\sum_{0}^{N} w_{k}(x) \varepsilon^{k}\right)
$$

для функции $q(x, \varepsilon)$ почти однородную начальную задачу:

$$
\varepsilon \frac{d q}{d x}=A(x, \varepsilon) q+\varepsilon^{N+1} b_{1}(x, \varepsilon), \quad q(0, \varepsilon)=\alpha-w_{(N)}(0, \varepsilon) \equiv \beta(\varepsilon)
$$

где регулярная по $\varepsilon$ векторная функция $w_{(N)}(x, \varepsilon)$ однозначно определяется (при подстановке ее в уравнение $(1))$ из соотношений

$$
0=A_{0}(x) w_{0}(x)+f(x), \quad \frac{d w_{k-1}}{d x}=A_{0}(x) w_{k}(x)+\cdots+A_{k}(x) w_{0}(x) \quad(k=\overline{1, N})
$$

при этом

$$
\left\|w_{k}^{(p)}(x)\right\| \leqslant C_{1 k p}, \quad\left(k=\overline{0, N}, \quad p=\overline{0, N+1}, \quad x \in \mathbb{R}^{+}\right) .
$$

Введем для произвольной матрицы $A=\left\{a_{j k}\right\}_{1}^{n}$ обозначения

$$
\bar{A}=\operatorname{diag}\left\{a_{11}, \ldots, a_{n n}\right\}, \quad \overline{\bar{A}}=A-\bar{A},
$$

необходимые для дальнейшего изложения.

ТЕОрема 1. Пусть для задачи (1) выполнены условия (2), (3), а также неравенство (5), спектр $\left\{\lambda_{0 j}(x)\right\}_{1}^{n}$ матрицы $A_{0}(x)$ удовлетворяет неравенствам

$$
\operatorname{Re} \lambda_{0 j}(x) \leqslant-\delta_{0}<0 \quad\left(j=\overline{1, n}, \quad x \in \mathbb{R}^{+}\right)
$$

кроме того, для матрии, $S_{0}(x), \Lambda_{0}(x), B_{k}(x)(k \geqslant 1)$, где

$$
\begin{gathered}
S_{0}^{-1}(x) A_{0}(x) S_{0}(x)=\Lambda_{0}(x)=\operatorname{diag}\left\{\lambda_{01}(x), \ldots, \lambda_{0 n}(x)\right\} \\
B(x, \varepsilon)=S_{0}^{-1}(x)\left(A(x, \varepsilon) S_{0}(x)-\varepsilon \frac{d S_{0}}{d x}\right)=\Lambda_{0}(x)+\sum_{1}^{\infty} B_{k}(x) \varepsilon^{k}
\end{gathered}
$$

справедливы оченки

$$
\begin{aligned}
& \left\|S_{0}(x)\right\| \leqslant C_{0}, \quad\left\|B_{q}^{(p)}(x)\right\| \leqslant C_{q p}, \quad\left\|\left(\frac{B_{q}(x)}{\sigma_{j k}(x)}\right)^{(p)}\right\| \leqslant C_{q p} \\
& \left(q \geqslant 1, \quad p=\overline{0, N+1}, \quad j \neq k, \quad j, k=\overline{1, n}, \quad x \in \mathbb{R}^{+}\right) .
\end{aligned}
$$


Тогда при достаточно мальх $\varepsilon>0$ и любых $x \in \mathbb{R}^{+}$существует единственное $u$ ограниченное при $\varepsilon \rightarrow+0$ решение $y(x, \varepsilon)$ задачи (1), имеющее асимптотическое представление

$$
\begin{aligned}
& y(x, \varepsilon) \sim Y_{(N)}(x, \varepsilon) \\
&=S_{0}(x)\left(E+\sum_{1}^{N} \overline{\bar{H}}_{k}(x) \varepsilon^{k}\right) \exp \left(\frac{1}{\varepsilon} \int_{0}^{x} \Lambda_{(N)}(t, \varepsilon) d t\right)+w_{(N)}(x, \varepsilon) \\
&\left(\Lambda_{(N)}(x, \varepsilon)=\sum_{0}^{N} \Lambda_{k}(x) \varepsilon^{k}\right)
\end{aligned}
$$

(матричные функиии $\overline{\bar{H}}_{k}(x)$ и $\Lambda_{k}(x)(k=\overline{1, N})$ однозначно определяются в ходе проведения алгоритма), причем на всей полуоси $\mathbb{R}^{+}$справедлива оченка

$$
\left\|y(x, \varepsilon)-Y_{(N)}(x, \varepsilon)\right\| \leqslant K \varepsilon^{N+1},
$$

где постоянная $K$ не зависит от $\varepsilon$.

ДокаЗАТЕЛьСтво. Для доказательства теоремы (с учетом проделанных преобразований) достаточно рассмотреть задачу (4), которая после невырожденной при достаточно малых $|\varepsilon|$ замены

$$
q(x, \varepsilon)=S_{0}(x)\left(E+\varepsilon \overline{\bar{H}}_{1}(x)\right) z
$$

принимает более простой вид:

$$
\begin{gathered}
\varepsilon \frac{d z}{d x}=\left(\Lambda_{(1)}(x, \varepsilon)+\varepsilon^{2} G_{2}(x, \varepsilon)\right) z+\varepsilon^{N+1} b_{2}(x, \varepsilon), \quad z(0, \varepsilon)=\mu(\varepsilon) \\
\left(\Lambda_{(1)}(x, \varepsilon)=\sum_{0}^{1} \Lambda_{k}(x) \varepsilon^{k}, \quad\left\|G_{2}\right\|,\left\|b_{2}\right\| \leqslant C\right),
\end{gathered}
$$

матрицы $\overline{\bar{H}}_{1}(x)$ и $\Lambda_{1}(x)$ определяются равенством

$$
\begin{gathered}
\Lambda_{0}(x) \overline{\bar{H}}_{1}(x)-\overline{\bar{H}}_{1}(x) \Lambda_{0}(x)=\Lambda_{1}(x)-B_{1}(x), \\
\Lambda_{1}(x)=\bar{B}_{1}(x), \quad \Lambda_{0}(x) \overline{\bar{H}}_{1}(x)-\overline{\bar{H}}_{1}(x) \Lambda_{0}(x)=-\overline{\bar{B}}_{1}(x),
\end{gathered}
$$

позволяя перейти к эквивалентному интегральному уравнению

$$
z=\Phi_{(1)}(x, \varepsilon)\left(\mu(\varepsilon)+\int_{0}^{x} \Phi_{(1)}^{-1}(t, \varepsilon)\left(\varepsilon G_{2}(t, \varepsilon) z+\varepsilon^{N} b_{2}(t, \varepsilon)\right) d t\right),
$$

где

$$
\begin{gathered}
\Phi_{(1)}(x, \varepsilon)=\exp \left(\frac{1}{\varepsilon} \int_{0}^{x} \Lambda_{(1)}(t, \varepsilon) d t\right), \\
\left\|\Phi_{(1)}(t, \varepsilon)\right\| \leqslant C_{1} \quad\left(x \in \mathbb{R}^{+}, \quad 0<\varepsilon \leqslant \varepsilon_{1}<\varepsilon_{0}\right) .
\end{gathered}
$$


При этом для любого фиксированного $x_{1} \in \mathbb{R}^{+}$в условиях теоремы справедливы неравенства

$$
\begin{gathered}
\|z\| \leqslant C_{0}+\varepsilon C_{1} \max _{\left[0, x_{1}\right]}\|z(x, \varepsilon)\|+\varepsilon^{N+1} C_{2}, \\
\max _{\left[0, x_{1}\right]}\|z(x, \varepsilon)\| \leqslant \frac{C_{0}+\varepsilon^{N} C_{2}}{1-\varepsilon C_{1}} \leqslant C, \quad \max _{\mathbb{R}^{+}}\|z(x, \varepsilon)\| \leqslant C,
\end{gathered}
$$

что и доказьвает существование единственного и ограниченного при $\varepsilon \rightarrow+0$ и любом $x \in \mathbb{R}^{+}$решении задачи (9), т.е. и исходной задачи (1). Покажем далее, что решение $Z_{0}(x, \varepsilon)$ однородной $\left(b_{2} \equiv 0\right)$ задачи $(9)$ может быть записано в виде

$$
z_{0}(x, \varepsilon)=H(x, \varepsilon) \exp \left(\frac{1}{\varepsilon} \int_{0}^{x} \Lambda(t, \varepsilon) d t\right)
$$

где неизвестные пока матричные функции

$$
H(x, \varepsilon)=E+\sum_{1}^{\infty} \overline{\bar{H}}_{k}(x) \varepsilon^{k} \quad \text { и } \quad \Lambda(x, \varepsilon)=\sum_{0}^{\infty} \Lambda_{k}(x) \varepsilon^{k}
$$

удовлетворяют уравнению

$$
\varepsilon \frac{d H}{d x}=B(x, \varepsilon) H-H \Lambda(x, \varepsilon),
$$

которое может быть получено после подстановки (10) в (9).

Приравнивая в (11) коэффициенты при одинаковых степенях $\varepsilon$, получим простые рекуррентные соотношения для последовательного и однозначного определения всех матриц $\overline{\bar{H}}_{k}(x)$ и $\Lambda_{k}(x)$ (аргументы опущены):

$$
\begin{gathered}
\Lambda_{0} \overline{\bar{H}}_{k}-\overline{\bar{H}}_{k} \Lambda_{0}=\Lambda_{k}-P_{k} \quad(k \geqslant 1), \\
P_{1}=B_{1}, \quad P_{k}=B_{k}+\sum_{j=1}^{k-1}\left(B_{j} \overline{\bar{H}}_{k-j}-\overline{\bar{H}}_{k-j} \Lambda_{j}\right)-\frac{d \overline{\bar{H}}_{k-1}}{d x} \quad(k \geqslant 2),
\end{gathered}
$$

при этом $\Lambda_{k}(x)=\bar{B}_{k}(x)$, а бездиагональные матрицы $\overline{\bar{H}}_{k}(x)$ удовлетворяют простым алгебраическим уравнениям

$$
\begin{gathered}
\Lambda_{0} \overline{\bar{H}}_{k}-\overline{\bar{H}}_{k} \Lambda_{0}=-\overline{\bar{P}}_{k}, \\
\left\|\Lambda_{k}(x)\right\|,\left\|\overline{\bar{H}}_{k}(x)\right\| \leqslant C_{k} \quad\left(k \geqslant 1, \quad x \in \mathbb{R}^{+}\right),
\end{gathered}
$$

решения которых не вызьвает затруднений. Покажем далее, что “усеченньй вектор"

$$
z_{(N)}(x, \varepsilon)=H_{(N)}(x, \varepsilon) \exp \left(\frac{1}{\varepsilon} \int_{0}^{x} \Lambda_{(N)}(t, \varepsilon) d t\right) C(\varepsilon),
$$

где

$$
H_{(N)}(x, \varepsilon)=E+\sum_{1}^{N} \overline{\bar{H}}_{k}(x) \varepsilon^{k}, \quad \Lambda_{(N)}(x, \varepsilon)=\sum_{0}^{N} \Lambda_{k}(x) \varepsilon^{k}
$$


будет удовлетворять при достаточно малых $\varepsilon>0$ задаче $(9)$ с точностью $O\left(\varepsilon^{N+1}\right)$. Запишем первые $N$ соотношений (12) (аргументы опущены)

$$
\begin{gathered}
0=\Lambda_{0} \overline{\bar{H}}_{1}-\overline{\bar{H}}_{1} \Lambda_{0}+B_{1}-\Lambda_{1}, \\
\frac{d \overline{\bar{H}}_{k-1}}{d x}=\Lambda_{0} \overline{\bar{H}}_{k}-\overline{\bar{H}}_{k} \Lambda_{0}+B_{k}+\sum_{j=1}^{k-1}\left(B_{j} \overline{\bar{H}}_{k-j}-\overline{\bar{H}}_{k-j} \Lambda_{j}\right)-\Lambda_{k} \quad(k=\overline{1, N}) .
\end{gathered}
$$

Сложив их (после умножения на соответствуюшие степени $\varepsilon$ ), получим дифференциальное матричное уравнение

$$
\varepsilon \frac{d H_{(N)}}{d x}=B(x, \varepsilon) H_{(N)}-H_{(N)} \Lambda_{(N)}(x, \varepsilon)+\varepsilon^{N+1} G_{3}(x, \varepsilon),
$$

которое после умножения справа на вектор

$$
\begin{gathered}
\Phi_{(N)}(x, \varepsilon) C(\varepsilon)=\exp \left(\frac{1}{\varepsilon} \int_{0}^{x} \Lambda_{(N)}(t, \varepsilon) d t\right) C(\varepsilon) \\
\left(\left\|\Phi_{(N)}(x, \varepsilon)\right\| \leqslant C_{N}, \quad\left\|G_{3}(x, \varepsilon)\right\| \leqslant K_{3}, \quad x \in \mathbb{R}^{+}, \quad 0<\varepsilon \leqslant \varepsilon_{1}<\varepsilon_{0}\right),
\end{gathered}
$$

дает возможность записать задачу для усеченного вектора $z_{(N)}(x, \varepsilon)$ :

$$
\begin{gathered}
\varepsilon \frac{d z_{(N)}}{d x}=\left(\Lambda_{(1)}(x, \varepsilon)+\varepsilon^{2} G_{2}(x, \varepsilon)\right) z_{(N)}+\varepsilon^{N+1} b_{3}(x, \varepsilon), \\
z_{(N)}(0, \varepsilon)=\mu(\varepsilon)+O\left(\varepsilon^{N+1}\right) \quad\left(\left\|b_{3}(x, \varepsilon)\right\| \leqslant K_{31}\right),
\end{gathered}
$$

и аналогичную задачу для усеченного вектора $q_{(N)}(x, \varepsilon)$ :

$$
\begin{gathered}
\varepsilon \frac{d q_{(N)}}{d x}=A(x, \varepsilon) q_{(N)}+\varepsilon^{N+1} b_{4}(x, \varepsilon), \\
q_{(N)}(0, \varepsilon)=\beta(\varepsilon)+O\left(\varepsilon^{N+1}\right) \quad\left(\left\|b_{4}(x, \varepsilon)\right\| \leqslant K_{4}\right) .
\end{gathered}
$$

Это означает, что векторы $z_{(N)}(x, \varepsilon)$ и $q_{(N)}(x, \varepsilon)$ удовлетворяют соответствующим задачам с точностью $O\left(\varepsilon^{N+1}\right)$.

Представим решение задачи (4) в виде

$$
q(x, \varepsilon)=q_{(N)}(x, \varepsilon)+\varepsilon^{N+1} v(x, \varepsilon) .
$$

При этом функция $v(x, \varepsilon)$ будет удовлетворять задаче

$$
\begin{gathered}
\varepsilon \frac{d v}{d x}=A(x, \varepsilon) v+b_{5}(x, \varepsilon), \\
v(0, \varepsilon)=O\left(\varepsilon^{N+1}\right) \quad\left(\left\|b_{5}(x, \varepsilon)\right\| \leqslant K_{5}\right),
\end{gathered}
$$

аналогичной исходной задаче (1), имеющей (по доказанному выше) равномерно ограниченное на всей полуоси $\mathbb{R}^{+}$решение при достаточно малых $\varepsilon>0$, что и доказывает (с учетом всех проделанных замен) справедливость оценки (8), завершая тем самым доказательство теоремы 1. 
ЗАмЕчАНИЕ. В асимптотическом представлении (7) некоторые из интегралов могут оказаться ограниченными при $x \rightarrow+\infty$, т.е. существуют такие номера $p(1 \leqslant p \leqslant N)$, для которых справедливы неравенства

$$
\left\|\int_{0}^{x} \Lambda_{p}(t) d t\right\| \leqslant C_{p}
$$

Это позволяет соответствующие экспоненты разложить в регулярные по $\varepsilon$ ряды и выделить сингулярности искомого решения в чистом виде, при этом асимптотическое представление решения $y(x, \varepsilon)$ принимает вид

$$
Y_{(N)}(x, \varepsilon)=S_{0}(x) \sum_{0}^{N} S_{k}(x) \varepsilon^{k} \exp \left(\frac{1}{\varepsilon} \int_{0}^{x} \sum_{0}^{N} \Lambda_{k}(t) \varepsilon^{k} d t\right)+w_{(N)}(x, \varepsilon),
$$

где сумма $\sum^{\prime}$ содержит только неограниченные при $x \rightarrow+\infty$ интегралы.

Аналогичный результат имеет место и в критическом случае, когда точки спектра матрицы $A_{0}(x)$ могут касаться мнимой оси.

ТЕОрема 2. Пусть для задачи (1) выполнены условия (2), (3), (5), (6) и, кроме того, спектр $\left\{\lambda_{0 j}(x)\right\}_{1}^{n}$ матрицы $A_{0}(x)$ удовлетворяет неравенствам

$$
\begin{array}{ll}
\operatorname{Re} \lambda_{0 k}(x) \leqslant 0 & \left(k=\overline{1, p}, \quad x \in \mathbb{R}^{+}\right), \\
\operatorname{Re} \lambda_{0 j}(x) \leqslant-\delta_{0}<0 & \left(j=\overline{p+1, n}, \quad x \in \mathbb{R}^{+}\right),
\end{array}
$$

при этом вспомогательная матрица

$$
\Lambda_{1}(x)=\bar{B}_{1}(x)=\operatorname{diag}\left\{\lambda_{11}(x), \ldots, \lambda_{1 n}(x)\right\}
$$

имеет спектр $\left\{\lambda_{1 j}(x)\right\}_{1}^{n}$, для которого справедливы соотношения

$$
\operatorname{Re} \lambda_{1 k}(x) \leqslant-\delta_{1}<0 \quad\left(k=\overline{1, p}, \quad x \in \mathbb{R}^{+}\right) .
$$

Тогда задача (1) имеет единственное и ограниченное $n р и \varepsilon \rightarrow+0$ и любых $x \in \mathbb{R}^{+}$ решение $y(x, \varepsilon)$, асимптотическое представление которого имеет вид (7), причем на всей полуоси справедлива оценка (8), где постоянная $K$ не зависит от $\varepsilon$.

Доказательство теоремы 2 в том числе и построение асимптотического представления решения задачи (1) вида (7) в этом случае во многом аналогично доказательству теоремы 1 и нами опускается.

ЗАмЕчАнИЕ 1. Остается в силе замечание к теореме 1.

ЗАмечание 2 . Теорема 2 имеет место и в случае, когда спектр $\left\{\lambda_{j(N)}(x, \varepsilon)\right\}_{1}^{n}$ вспомогательной матрицы

$$
\Lambda_{(N)}(x, \varepsilon)=\sum_{0}^{N} \Lambda_{k}(x) \varepsilon^{k}=\operatorname{diag}\left\{\lambda_{1(N)}(x, \varepsilon), \ldots, \lambda_{n(N)}(x, \varepsilon)\right\}
$$

при достаточно малых $\varepsilon>0$ удовлетворяет неравенствам

$$
\operatorname{Re} \lambda_{j(N)}(x, \varepsilon) \leqslant-\varepsilon^{k_{j}} \delta<0 \quad\left(j=\overline{1, n}, \quad 0 \leqslant k_{j} \leqslant N, \quad x \in \mathbb{R}^{+}\right) .
$$

ЗАмЕчАниЕ 3 . При наличии у матрицы $A_{0}(x)$ тождественно кратных точек спектра асимптотическое представление решения задачи (1) может в общем случае содержать дробные степени малого параметра $\varepsilon$, что следует из результатов работ [9], [10]. 


\section{СПИСОК ЦИТИРОВАННОЙ ЛИТЕРАТУРЫ}

[1] Ломов С. А. Введение в общую теорию сингулярных возмущений. М.: Наука, 1981.

[2] Федорюк М.В.Асимптотические методы в теории обыкновенных линейных дифференциальных уравнений // Матем. сб. 1969. Т. 79(121). № 4. С. 477-516.

[3] Федорюк М. В. Асимптотические методы в анализе // Итоги науки и техн. Соврем. пробл. матем. Фундамент. направления. Т. 13. М.: ВИНИТИ, 1986. С. 93-210.

[4] Фёдоров Ю.С. Построение фундаментальной матрицы сингулярно возмущенной системы уравнений в некомпактной области // Школа "Современные методы в теории краевых задач". Тезисы докл. Воронеж, 1992. С. 111.

[5] Фёдоров Ю. С. Асимптотическое интегрирование краевой задачи на полуоси // Тр. МЭИ. 1987. № 141. С. 99-102.

[6] Коняев Ю.А. Общий подход к асимптотическому интегрированию сингулярно возмущенных начальных и краевых задач для систем обыкновенных дифференциальньх уравнений // Дифференц. уравнения. 1984. Т. 20. №11. С. 1999-2003.

[7] Коняев Ю.А. Исследования некоторых классов регулярных и сингулярных краевых задач // Матем. заметки. 1992. Т. 51. № 2. С. 149-151.

[8] Коняев Ю.А. Конструктивные методы многоточечных краевых задач // Изв. вузов. Матем. 1992. № 2. С. 57-61.

[9] Коняев Ю. А. О новом подходе к исследованию линейных сингулярно возмущенных задач при наличии тождественно кратных и мнимых точек спектра // Дифференц. уравнения. 1985. T. 21. № 10. С. 1811-1814.

[10] Коняев Ю. А. Об одном методе исследования некоторых задач теории возмущений // Матем. сб. 1993. № 12. С. 133-144. 